PROCEEDINGS OF THE

AMERICAN MATHEMATICAL SOCIETY

Volume 131, Number 8, Pages 2325-2328

S 0002-9939(02)06823-5

Article electronically published on November 14, 2002

\title{
BELL REPRESENTATIONS OF FINITELY CONNECTED PLANAR DOMAINS
}

\author{
MOONJA JEONG AND MASAHIKO TANIGUCHI \\ (Communicated by Mei-Chi Shaw)
}

\begin{abstract}
In this paper, we solve a conjecture of S. Bell (1992) affirmatively. Actually, we prove that every non-degenerate $n$-connected planar domain $\Omega$, where $n>1$ is representable as $\Omega=\{|f|<1\}$ with a suitable rational function $f$ of degree $n$. This result is considered as a natural generalization of the classical Riemann mapping theorem for simply connected planar domains.
\end{abstract}

\section{INTRODUCTION AND THE MAIN THEOREM}

Recently, S. Bell posed the following problem (B1] and [B2]).

Problem 1.1. Can every non-degenerate $n$-connected planar domain with $n>1$ be mapped biholomorphically onto a domain of the form

$$
\left\{\left|z+\sum_{k=1}^{n-1} \frac{a_{k}}{z-b_{k}}\right|<r\right\}
$$

with complex numbers $a_{k}$ and $b_{k}$, and a positive $r$ ?

Here and in the sequel, a non-degenerate $n$-connected planar domain is a subdomain $\Omega$ of the Riemann sphere $\hat{\mathbb{C}}$ such that $\hat{\mathbb{C}}-\Omega$ consists of exactly $n$ connected components each of which contains more than one point. In this note, we solve this problem affirmatively. Actually, we give a proof of the following assertion.

Theorem 1.2. Every non-degenerate n-connected planar domain with $n>1$ is mapped biholomorphically onto a domain defined by

$$
\left\{\left|z+\sum_{k=1}^{n-1} \frac{a_{k}}{z-b_{k}}\right|<1\right\}
$$

with suitable complex numbers $a_{k}$ and $b_{k}$.

Recall that every domain defined as in Theorem 1.2 has algebraic kernel functions. See Theorem 4.4 in B1]. This is one of the reasons why we consider such domains.

Received by the editors March 15, 2002.

2000 Mathematics Subject Classification. Primary 32G10, 32G15; Secondary 30C20, 30F60.

Key words and phrases. Conformal representation, Ahlfors maps.

The second author was supported in part by Grant-in-Aid for Scientific Research (B)(2) 200113440047 . 
Remark 1.3. It is well known (cf. for instance [IT]) that the reduced Teichmüller space $T(\Omega)$ of a non-degenerate $n$-connected planar domain $\Omega$ can be identified with the Fricke space of a Fuchsian model $G$ of $\Omega$. Since $G$ is a free real Möbius group with $n-1$ hyperbolic generators, $T(\Omega)$ is real ( $3 n-6)$-dimensional.

Such a Bell representation as in Theorem 1.2 contains $2 n-2$ complex, i.e. $4 n-4$ real, parameters. The reason why we need many more number of parameters in a Bell representation than Teichmüller parameters for $T(\Omega)$ is that every Bell representation of a domain is actually associated with an $n$-sheeted branched covering of the unit disk by $\Omega$.

Remark 1.4. Such a space as $H_{0, n}$ consisting of all branched coverings of $\hat{\mathbb{C}}$ induced by rational functions of degree $n$ with $n>1$ is called a Hurwitz space $([\mathbb{N}])$. This space $H_{0, n}$ is parametrized by $2 n-2$ critical values (the images of critical points), and hence is complex $(2 n-2)$-dimensional.

Actually, we show that every Ahlfors map on $\Omega$ can be considered as the restriction of a rational function of degree $n$ to a suitable domain which can be identified with $\Omega$. On the other hand, the set of all branched coverings of $\hat{\mathbb{C}}$ induced from Bell representations can be considered as a subdomain of $H_{0, n}$. Thus to solve the following problem would be interesting.

Problem 1.5. Find the sublocus $A_{0, n}$ of $H_{0, n}$ which corresponds to the set of all Bell representations of non-degenerate $n$-connected planar domains such that the restrictions of the associated rational functions give Ahlfors maps.

The authors also thank the referee for his/her valuable suggestions and comments.

\section{Proof of Theorem 1.2}

First let a non-degenerate $n$-connected planar domain $\Omega$ be given. Then by using the classical Riemann mapping theorem $n$ times if necessary, we can assume that the boundary of $\Omega$ consists of exactly $n$ smooth simple closed curves. Fix a point $a$ in $\Omega$, and let $f_{a}$ be the Ahlfors map associated to the pair $(\Omega, a)$. Here for the definition and properties of the Ahlfors maps, see for instance, $[\mathrm{B}$. In particular, $f_{a}$ maps $\Omega$ properly and holomorphically onto the unit disk $U$. Moreover, $f_{a}$ can be extended to a continuous map of the closure $\bar{\Omega}$ of $\Omega$ onto the closed unit disk so that every component $\gamma_{j}$ of the boundary of $\Omega$, where $j=1, \cdots, n$, is mapped homeomorphically onto the unit circle.

Lemma 2.1. There is a compact Riemann surface $R$ (without boundary) of genus 0 and a holomorphic injection $\iota$ of $\Omega$ into $R$ such that

$$
f_{a} \circ \iota^{-1}
$$

can be extended to a meromorphic function, say $F$, on $R$.

Proof. Since there are only a finite number of zeros of $f_{a}^{\prime}$, there is a positive constant $\rho$ such that $\rho<1$ and that

$$
D=\{\rho<|\zeta|<1\}
$$

where $\zeta$ is the complex coordinate on the target plane of the map $f_{a}$, and contains no critical values (i.e. no images of the zeros of $f_{a}^{\prime}$ by $f_{a}$ ). Hence every component 
$W_{j}$, where $j=1, \cdots, n$, of $f_{a}^{-1}(D)$ is mapped biholomorphically onto $D$ by the restriction $\left.f_{a}\right|_{W_{j}}$, of $f_{a}$ to $W_{j}$.

Now we construct a compact Riemann surface $R$ by using the Ahlfors map $f_{a}$ to attach disks to the exterior of $\Omega$ along each boundary curve. More precisely, we consider the disjoint union $\mathbf{R}$ of $\Omega$ and $n$ copies $V_{j}(j=1, \cdots, n)$ of

$$
V=\{\rho<|\zeta|\} \cup\{\infty\} .
$$

Identify every subdomain $W_{j}$ of $\Omega$ with the subdomain $D_{j}$ of $V_{j}$ corresponding to $D$ by the biholmorphic map corresponding to $\left.f_{a}\right|_{W_{j}}$. Then the resulting set, which we denote by $R=\mathbf{R} / f_{a}$, has a natural complex structure induced from those on $\Omega$ and on every $V_{j}$, and hence is a Riemann surface. Here the natural inclusion map $\iota$ of $\Omega$ into $R$ is a holomorphic injection, and using the complex coordinate $\zeta_{j}$ on the copy $V_{j}$ corresponding to $\zeta$ on $V$, we have

$$
f_{a} \circ \iota^{-1}\left(\zeta_{j}\right)=\zeta
$$

on $D_{j}$ by the definition.

Now, since topologically $R$ is obtained from $\Omega$ by attaching a disk along each boundary curve of $\Omega, R$ is a simply connected compact Riemann surface without boundary, and hence in particular, is of genus 0 . Also we can extend $F=f_{a} \circ \iota^{-1}$ to a meromorphic function on the whole $R$ by setting $F\left(\zeta_{j}\right)=\zeta$ and $F(\infty)=\infty$ on the whole $V_{j}$ for every $j$.

Here the following uniformization theorem (which is also called the generalized Riemann mapping theorem) is classical and well-known. As references, we cite for instance [FK] and [IT].

Proposition 2.2 (Klein, Koebe and Poincaré). Every simply connected Riemann surface is mapped biholomorphically onto one of

- the unit disk $U$,

- the complex plane $\mathbb{C}$, and

- the Riemann sphere $\hat{\mathbb{C}}$.

Corollary 2.3. There is a biholomorphic map $h$ of the above Riemann surface $R$ onto the Riemann sphere $\hat{\mathbb{C}}$, and hence $F \circ h^{-1}$ is a rational function.

Proof. Since $R$ is compact, $R$ is mapped by a biholomorphic map $h$ onto the Riemann sphere. Set $f=F \circ h^{-1}$. Then $f$ is meromorphic on the whole $\hat{\mathbb{C}}$, which implies that $f$ is a rational function.

Here and in the sequel, we may assume that

$$
f(\infty)=\infty
$$

by applying to $f$ the pre-composition of a Möbius transformation $S$ which sends $\infty$ to a pole of $f$, i.e. by replacing $h$ by $S^{-1} \circ h$, if necessary.

Lemma 2.4. Let $w$ be the complex variable of the above rational function $f$. Then $f$ has the following partial fraction decomposition:

$$
f(w)=C w+D+\sum_{k=1}^{n-1} \frac{A_{k}}{w-B_{k}} .
$$

Here $A_{k}, B_{k}, C$ and $D$ are complex constants, every $A_{k}$ and $C$ are non-zero, and $\left\{B_{k}\right\}$ are mutually distinct. 
Proof. Since $f$ has exactly $n$ simple poles, as is seen from the construction, and one of them is $\infty$ by the above assumption, $f$ is of degree exactly $n$ and has $n-1$ finite, mutually distinct, simple poles, say $B_{1}, \cdots, B_{n-1}$. Hence we can write $f(w)$ as

$$
f(w)=\frac{P(w)}{Q(w)}
$$

with polynomials $P(w)$ of degree exactly $n$ and

$$
Q(w)=\left(w-B_{1}\right) \cdots\left(w-B_{n-1}\right) .
$$

Thus it is easy to see that the partial fraction decomposition of $f$ is as claimed.

Proof of Theorem 1.2. We replace the complex variable $w$ of $f$ by

$$
z=T(w)=C w+D
$$

by applying to $f$ the precomposition by an affine transformation $T$. Further set

$$
a_{k}=C A_{k}, \quad b_{k}=C B_{k}+D
$$

for every $k$. Then we conclude that

$$
f \circ T^{-1}(z)=z+\sum_{k=1}^{n-1} \frac{a_{k}}{z-b_{k}} .
$$

Thus the Bell representation

$$
\left\{\left|z+\sum_{k=1}^{n-1} \frac{a_{k}}{z-b_{k}}\right|<1\right\}
$$

is the subdomain

$$
\left\{\left|f \circ T^{-1}(z)\right|<1\right\}=\left\{\left|F \circ h^{-1} \circ T^{-1}(z)\right|<1\right\}=(T \circ h \circ \iota)(\Omega)
$$

of $\hat{\mathbb{C}}$, which is mapped biholomorphically onto $\Omega$ by the holomorphic injection $(T \circ h \circ \iota)^{-1}$.

\section{REFERENCES}

[B] S. R. Bell, The Cauchy Transform, Potential Theory, and Conformal Mapping, CRC Press, Boca Raton, 1992. MR 94k:30013

[B1] S. R. Bell, Finitely generated function fields and complexity in potential theory in the plane, Duke Math. J., 98 (1999), 187-207. MR 2000i:30015

[B2] S. R. Bell, A Riemann surface attached to domains in the plane and complexity in potential theory, Houston J. Math., 26, (2000), 277-297. MR 2001m:30009

[FK] H. M. Farkas and I. Kra, Riemann Surfaces, Springer-Verlag, 1980. MR 82c:30067

[IT] Y. Imayoshi and M. Taniguchi, An Introduction to Teichmüller Spaces, Springer-Verlag, Tokyo, 1992. MR 94b:32031

[N] S. M. Natanzon, Hurwitz spaces, Topics on Riemann surfaces and Fuchsian Groups, LMS Lecture Notes, 287 (2001), 165-177.

Department of Mathematics, The University of Suwon, Suwon P.O. Box 77, KyungKIDO, 440-600, KOREA

E-mail address: mjeong@mail.suwon.ac.kr

Department of Mathematics, Graduate school of Science, Kyoto University, Kyoto 606, JAPAN

E-mail address: tanig@kusm.kyoto-u.ac.jp 